\title{
Structural Analysis and Improved Reactive Power Alignment for Secondary Voltage Control
}

\author{
$1^{\text {st }}$ Allal El Moubarek BOUZID \\ Ecole Centrale Nantes LS2N-CNRS \\ Nantes, France \\ Allal.El.Moubarek.Bouzid@1s2n.fr
}

\author{
$2^{\text {nd }}$ Bogdan MARINESCU \\ Ecole Centrale Nantes LS2N-CNRS \\ Nantes, France \\ Bogdan.Marinescu@ec-nantes.fr
}

\author{
$3^{\text {rd }}$ Guillaume DENIS \\ $R T E-R \& D$ \\ Paris, France \\ Guillaume.Denis@rte-france.com
}

\begin{abstract}
This paper proposes a new strategy for secondary voltage control which is easier to implement and improves the alignment of reactive power of generators The control objectives are revisited from both static and dynamic points of view using the internal model principle. A new alignment strategy for the reactive power generations is proposed along with robust dynamic control. The methodology is applied to interconnected power networks based on real data on EUROSTAG. The simulation results show the performance of the proposed methods to regulate the voltage on the pilot buses at the desired values.
\end{abstract}

Index Terms-Full state feedback, secondary voltage control, Linear Quadratic Integral, reactive power, pilot points.

\section{INTRODUCTION}

In power systems, voltage regulation plays an important role and its synthesis is a great challenge to ensure the safety of the whole electrical network [1], [2]. In fact, besides the commitment to the customers, Transmission Systems Operators (TSO) have to maintain grid voltage in specified limits which guarantee the operating safety and optimality. The voltage control is usually ensured by a hierarchical system with three levels: primary, secondary and tertiary voltage control (see, e.g., [3], [4], [5]). The primary voltage control is performed by local regulators, Automatic Voltage Regulators (AVR) and aims to fast regulate the terminal voltage of the generator. The grid is next shared into zones and, for each zone, a regional level called Secondary Voltage Control (SVC) adjusts and maintain the voltages at well-chosen buses inside the zone, called pilot-points [6], to desired values. This action is performed by computing the adjustment of AVR's set-points of each generator which participates to the SVC, its dynamics is slower than the one of AVRs (arround $2-3$ minutes). The tertiary voltage control performed at the dispatching center for the whole system is usually an optimal power-flow which determines an optimal voltage profile of the network according to safety and economic criteria. This strategy is now being extended to microgrids and to renewable energies power sources (see, e.g., [7], [8]). In most of the existing implementations, the SVC computes for each zone of the grid a signal representing the required reactive power level (e.g., [4]). This signal is used to adjust the AVR's set-points of each generator by a local unit reactive power control that regulates the reactive power generation. These two control loops are time decoupled in order to avoid interactions. However, this hierarchization suffers from a structural problem.
It provides a non minimal phase response which may lead also to a transient instability, as explained in [3]. Coordinated Secondary Voltage Control (CSVC) has been proposed to overcome these difficulties and to manage larger zones of the grid [3], [9], [10], [11]. An inter-plant voltage control was introduced for improving the voltage stability margin by coordinating reactive power reserves [12]. However, such solutions often lay on on-line (i.e., at each control sampling time) optimization of multi-variable quadratic function which is difficult to implement in real-time, especially when a lot of distant (PMU) measures have to be used [13]. Moreover, voltage and reactive power tracking objectives are mixed into a quadratic index to be minimized and this leads to uncertain reactive power alignment. In this paper, we first analyze the dynamic feasibility of the SVC objectives: regulate the voltage at a set of pilot-points and align the reactive power production of generating units proportionally to their capabilities. We next propose a robust approach to synthesize a multivariable proportional-integral controller able to achieve the two objectives in a robust coordinated way. The approach take advantage of both SVC and CSVC principles. The performances of this approach are compared to the classic SVC's ones. The paper is organized as follows: In Section II we recall the classic SVC and analyze their dynamics feasibility from a systemic point of view. The new SVC scheme with the design approach for synthesizing optimal gains is presented and analyzed in Section III. Numerical results from simulations of a real highvoltage power grid with EUROSTAG are given in Section IV before we conclude the work in Section V.

\section{SECONDARY VOLTAGE CONTROL}

As the SVC action is much slower (about 10 times) than the primary (AVR) transients, the local electric dynamics can be neglected and behavior of the network under the SVC action can be modeled by sensitivities matrices of the pilot-point voltage $V_{p p}$ and reactive powers $Q=\left[Q_{1}, . . Q_{n}\right]^{T}$ with respect to the terminal voltages of generators $V=\left[V_{1}, \ldots, V_{n}\right]^{T}$, where $n$ is the number of generators that participate to the voltage 


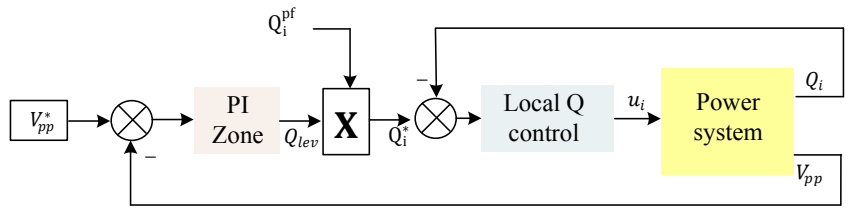

Figure 1: Block-diagram of classic SVC

control ${ }^{1}$ :

$$
\begin{aligned}
V_{p p} & =C_{v} V \\
Q & =C_{q} V .
\end{aligned}
$$

A dynamic model is obtained by considering a transfer function between the SVC control $U$ and the terminal voltage $V$ to take into account the primary voltage control dynamics of the generator $(\tau=1 s)$ :

$$
V=H(s) u, H(s)=1 /(1+s \tau) .
$$

From (1), (2) and (3) one can deduce the state-space form in which generator terminal voltage are chosen as state variables

$$
\begin{aligned}
\dot{x} & =A x+B u \\
{\left[\begin{array}{c}
V_{p p} \\
Q
\end{array}\right] } & =\left[\begin{array}{l}
C_{v} \\
C_{q}
\end{array}\right] x
\end{aligned}
$$

with $A=-\frac{1}{\tau} \operatorname{diag}\{1, \ldots, 1\} \in \mathbb{R}^{n \times n}$ and $B=$ $\frac{1}{\tau} \operatorname{diag}\{1, \ldots, 1\} \in \mathbb{R}^{n \times n}$ Notice that this modeling is general but, in this work we will consider zones with only one pilotpoint. Thus, $V_{p p}$ is here a scalar.

\section{A. Classic SVC}

The principle of the classic SVC is to compute for each zone a signal $Q_{l e v}$ representing the required reactive power level, through a proportional integral controller (6) which compares $V_{p p}$ to its set-point $V_{p p}^{*}$. This signal is used to adjust the AVR's set-points by the reactive control loop (7) which regulates the reactive power generation $Q_{i}$ in order to align its steady-state value with the ones of the others generating units, according to participation factors $Q_{i}^{p f}$ given for each generator. These two control loops are time-decoupled (local $Q$ loop faster than the reactive power level computation) in order to avoid interactions and facilitate implementation.

$$
\begin{aligned}
Q_{l e v} & =K_{P_{V}}\left(V_{p p}^{*}-V_{p p}\right)+K_{I_{V}} \int_{0}^{t}\left(V_{p p}^{*}-V_{p p}\right) d t \\
u_{i} & =K_{I_{Q}} \int_{0}^{t}\left(Q_{i}^{*}-Q_{i}\right) d t \\
Q_{i}^{*} & =Q_{l e v} Q_{i}^{p f}
\end{aligned}
$$

\footnotetext{
${ }^{1}$ In 1 and 2 the variables are variations between two operation points. As this model will be in what follows integrated into a linear dynamic one, notations are simplified to consider directly the variables and not their variations.
}

\section{B. Control objectives revisited}

The SVC should mainly

i) ensure pilot-point voltage,

$$
\lim _{t \rightarrow \infty} V_{p p}(t)=V_{p p}^{*} .
$$

ii) manage the reactive power generation of the groups which participate to SVC.

Objective i) has, in most cases, priority against objective ii). In the past, the latter objective was quantified in a global need in reactive power (linked to the voltage drop) of the region which is the level $Q_{l e v}$ in (8), shared next among the participating generators $Q_{i}^{*}$ by the use of local control loops (7). These loops have been proven to have dynamic interactions with the global voltage dynamics of the region which lead into non-minimal phase behavior. Indeed, the SVC hierarchization mentioned in the Introduction suffers from a structural problem. In fact, a stator voltage deep triggers both primary regulators and regional voltage control loop. The action of the primary regulators being faster, their response to maintain the voltage to the desired value is diminished by the reactive power control loop (7) which is driven by the voltage control loop (6). This unwanted action will last about several seconds until the reactive level of the zone changes. As shown in Fig 9 in case of a generator shut-down it provides a response in opposite direction of the desired response of the reactive power which may lead also to transient instability, as explained in [3].

To avoid this, new solutions were proposed like, in , e.g., [3], in which the local loops are suppressed but still a constant reactive power reference is proposed to be tracked by each generator. From the control point of view, this is not possible to be ensured along with item i) above. From the application point of view, it is not necessary to track precise pre-specified references for all the generators. It is sufficient to align the reactive power generations, i.e., to ensure that each generator participate in proportion to its capacity to the overall voltage regulation effort of the zone. This led us to revisit the SVC objectives formulation at both static and dynamic levels.

1) Static SVC objectives: Static means here in steady state, i.e., after extinction of all transients. To ensure objective i), the steady state pilot-point voltage should be $V_{p p}^{*}$. For the reactive power, one should first ask if the reactive power generations of all groups can be controlled. This is the case if an alignment on an average level of the zone defined as

$$
Q_{b a l}=\frac{\sum_{i=1}^{n} Q i}{\sum_{i=1}^{n} Q_{i}^{p f}}
$$

where $Q_{i}^{p f}$ is a (constant) participation factor of each generator is envisaged instead of constant and different setpoints as $Q_{i}^{*}$ in (8) for each reactive power generation.The predefined ratio $Q_{i}^{*}=\frac{Q_{i}}{Q_{i}^{p f}}$ should follow the average zone level eq. (10).

Lemma 2.1: Consider a zone of one pilot-point and $n$ generators and let $j \in\{1, \ldots, n\}$. 
if $Q_{i}^{*}=Q_{b a l}, i \in\{1, \ldots, n\}, i \neq j$ then

$$
Q_{j}^{*}=Q_{b a l}=0
$$

Proof: Rewrite (10) as : $Q_{b a l}=\frac{Q_{j}+Q_{b a l} \sum_{i \neq j} Q_{i}^{p f}}{Q_{j}^{p f}+\sum_{i \neq j} Q_{i}^{p f}}$ It follows that $Q_{b a l} Q_{j}^{p f}+Q_{b a l} \sum_{i \neq j} Q_{i}^{p f}=Q_{j}+Q_{b a l} \sum_{i \neq j} Q_{i}^{p f}$ from which $\frac{Q_{j}}{Q_{j}^{p f}}=Q_{b a l}$ thus $Q_{j}^{*}=Q_{b a l}$.

Lemma 2.1 shows that it is sufficient to regulate only $n-1$ reactive powers to align all the generators of a one pilot-point zone. This is an important result which will be exploited in the dynamic part of the control to choose the output to be regulated.

2) Dynamic SVC objectives: In order to align on $Q_{b a l}$ the production of generators which participate to the SVC, one should ensure the following tracking objectives $e_{1} \rightarrow 0$, $e_{2} \rightarrow 0$ when $t \longrightarrow+\infty$, where

$$
\left\{\begin{array}{l}
e_{1}=V_{p p}-V_{p p}^{*}=C_{v} x-V_{p p}^{*} \\
e_{2}=Q_{i}^{*}-Q_{b a l}=\left(C_{q}-C_{q}^{\prime}\right) x, i=1, \ldots, n
\end{array}\right.
$$

The number of variables to be tracked is again an important factor. Answer to this question is given using the internal model principle [14], [15] checked below for the SVC closed-loop. Consider for that a state-space representation like (5) along with a tracking objective

$$
e \rightarrow 0 \text { when } t \rightarrow+\infty, e=y-y_{\text {ref }},
$$

where $y=C x$ is a vector of chosen outputs and $y_{r e f}$ constant references for them.

Let $X=\left[\begin{array}{ll}\dot{x} & e\end{array}\right]^{T}$ an extended state-vector. The extendedstate system which corresponds to this is

$$
\dot{X}=\underbrace{\left[\begin{array}{cc}
A & 0 \\
C_{e} & 0
\end{array}\right]}_{\mathbb{A}} X+\underbrace{\left[\begin{array}{c}
B \\
0
\end{array}\right]}_{\mathbb{B}} \dot{u} .
$$

If $(\mathbb{A}, \mathbb{B})$ is stabilizable, there exist $K$ such that

$$
\dot{u}=-K X
$$

stabilizes the closed-loop, i.e., $X \rightarrow 0$ and, as a consequence, $e \rightarrow 0$.

Necessary and sufficient conditions for the existence of such a control are given by the internal model principle [14]:

Proposition 2.1: The pair $(\mathbb{A}, \mathbb{B})$ is stabilizable if, and only if, the following two conditions hold: $\left({ }^{*}\right) r \leq m ;\left({ }^{* *}\right) s=0$ is not an invariant zero of $\{A, B, C\}$.

Notice that condition $(*)$ limits the number of outputs which can be tracked. In the SVC context, objective (11) lead to $n+1$ variables to be tracked in a zone with $n$ generators. The number of actuators, i.e., $m$ in (**) is $n$ (dimension of $u$ in (4)). However, due to Lemma 2.1, steady-state alignment may be obtained in this context with only $n-1$ objectives type $e_{2}$ in (11). For this, the output $y$ for the control should be chosen $y=\left[\begin{array}{lllll}V_{p p} & Q_{i}-Q_{b a l} & Q_{j}-Q_{b a l} & \ldots & Q_{k}-Q_{b a l}\end{array}\right]^{T}$ where $Q_{i},{ }_{j} \ldots, k$ are $n-1$ reactive power generations chosen among the $n$ available to satisfy condition (**). Thus, $y_{r e f}=\left[\begin{array}{lllll}V_{p p}^{*} & 0 & 0 & \ldots & 0\end{array}\right]^{T}$ and condition $(*)$ is also satisfied.

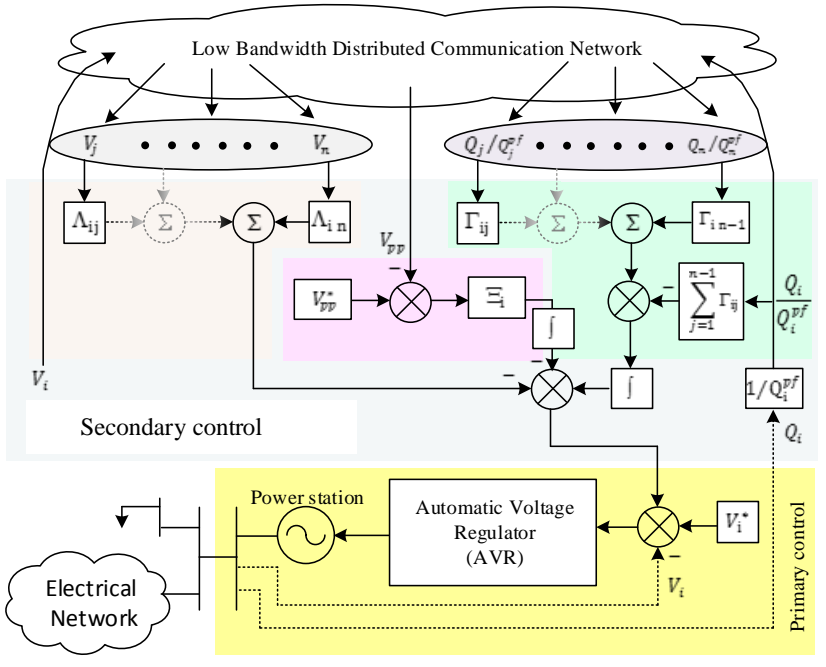

Figure 2: Block diagram of proposed control architecture

\section{NEW SVC CONTROL LAW}

Notice that the resulting control law (16) leads to a multivariable (i.e., matrix) Proportional-Integral (PI) control law

$$
u=-K_{P} x-K_{I} \int_{0}^{t} e d t
$$

which can be detailed for each generator

$u_{i}=-\sum_{i=1}^{n} \Lambda_{i} V_{i}-\int_{0}^{t} \Xi_{i}\left(V_{p p}^{*}-V_{p p}\right)-\int_{0}^{t} \sum_{j=1}^{n-1} \Gamma_{i j}\left(\frac{Q_{i}}{Q_{i}^{p f}}-\frac{Q_{j}}{Q_{j}^{p f}}\right)$

Matrices $\Lambda_{i j}, \Xi_{i}$ and $\Gamma_{i j}$ contain respectively the stator voltage, pilot-point and reactive power alignment gains for each generator. They are extracted from $K_{P}$ and $K_{I}$ of (15). A detailed schematic architecture of the new proposed secondary controller (16) is shown in Fig. 2.

Gains computation is the result of a pole placement in order to satisfy the decoupling between the secondary control loop and the primary control loop by choosing sufficiently large time constants without performance degradation and/or losing stability issues. For simplicity and as a first step in presentation, a LQR method [16] has been used at this stage. For the 4-generators example in Fig 3, with the choice $y=\left[\begin{array}{llll}V_{p p} & Q_{1} & Q_{2} & Q_{3}\end{array}\right]$ the resulting control, for example for generator 1, is:

$$
\begin{aligned}
u_{1} & =-0.0097 V_{1}+0.0012 V_{2}+0.0012 V_{3}-0.00052 V_{4} \\
& +0.011 \int_{0}^{t}\left(V_{p p}^{*}-V_{p p}\right) d t+0.00083 \int_{0}^{t}\left(Q_{b a l}-\frac{Q_{1}}{Q_{1}^{p f}}\right) d t \\
& -0.00016 \int_{0}^{t}\left(Q_{b a l}-\frac{Q_{2}}{Q_{2}^{p f}}\right) d t-0.00016 \int_{0}^{t}\left(Q_{b a l}-\frac{Q_{3}}{Q_{3}^{p f}}\right) d t
\end{aligned}
$$

Notice that, on the contrary of the classic SVC eq. (7), this control uses explicit voltage and reactive power information 


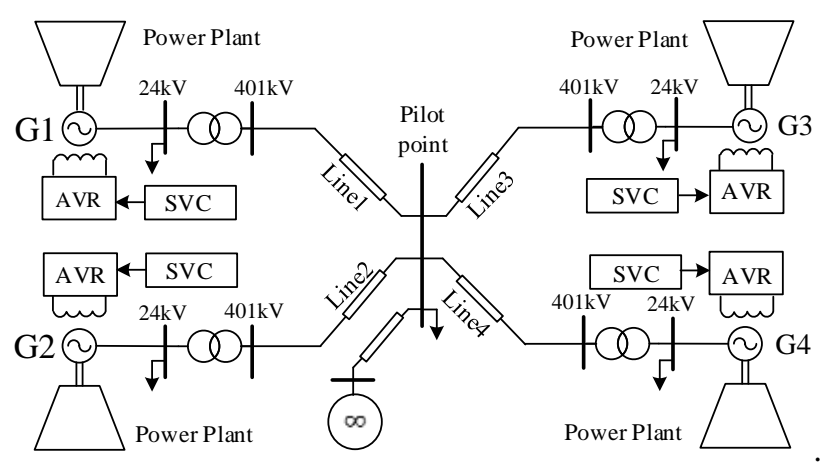

Figure 3: Schematic of the power grid setup

from all generators and this will provide enhanced robustness in case of disturbances and failures as shown in the next section. Also, this information is available as measurements, so no estimator is needed for implementation.

\section{Simulation Results}

Tests are presented for a 4-generators 4480 MVA power plant connected to a $401 \mathrm{kV}$ pilot-point if the french grid. The genarators are identical; modeled in full non linear detail along with their Automatic Voltage Regulator (AVR), governor, and protections using real data in simulation from RTE-France and implemented in EUROSTAG. The synthesis of the new SVC controller is carried out in MATLAB. The results are compared to the ones obtained with the classic SVC control (7).

\section{A. Study 1: Controller Performances}

1) Voltage Tracking Performance Assessment: As shown in Figure 4, the two controllers are synthesized to produce the
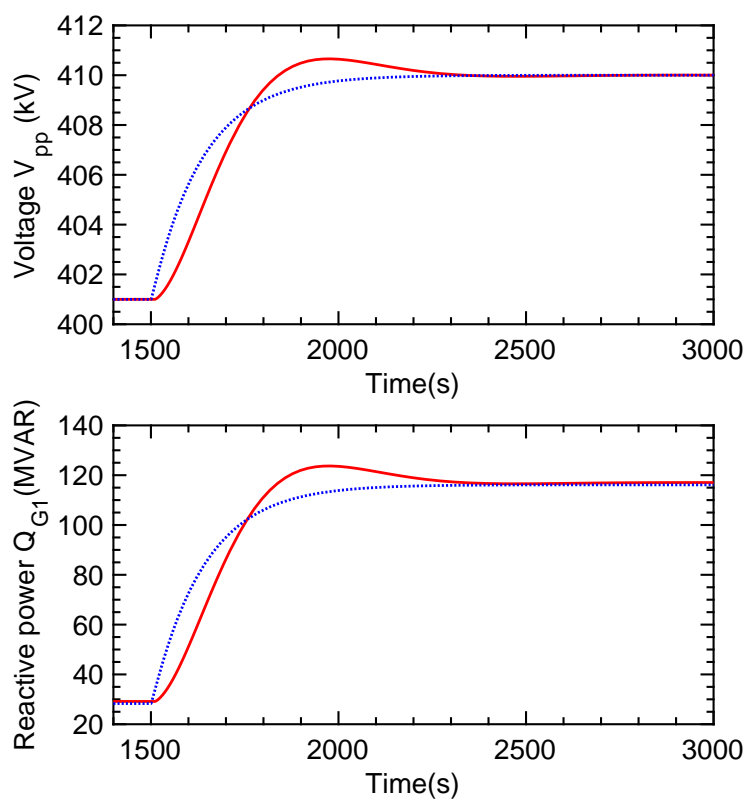

Figure 4: Performance of secondary voltage control considering voltage tracking (red : classic SVC, blue : new SVC) same time response of the closed loop in order to be able to compare their robustness. This dynamics corresponds to the usual SVC one, i.e, responses in about $5 \mathrm{~min}$. However, it can be seen that the voltage step response $V_{p p}^{*}=410 \mathrm{kV}$ at $t=1500 \mathrm{~s}$ of the classic SVC presents an overshoot with low rise time compared with the proposed controller.
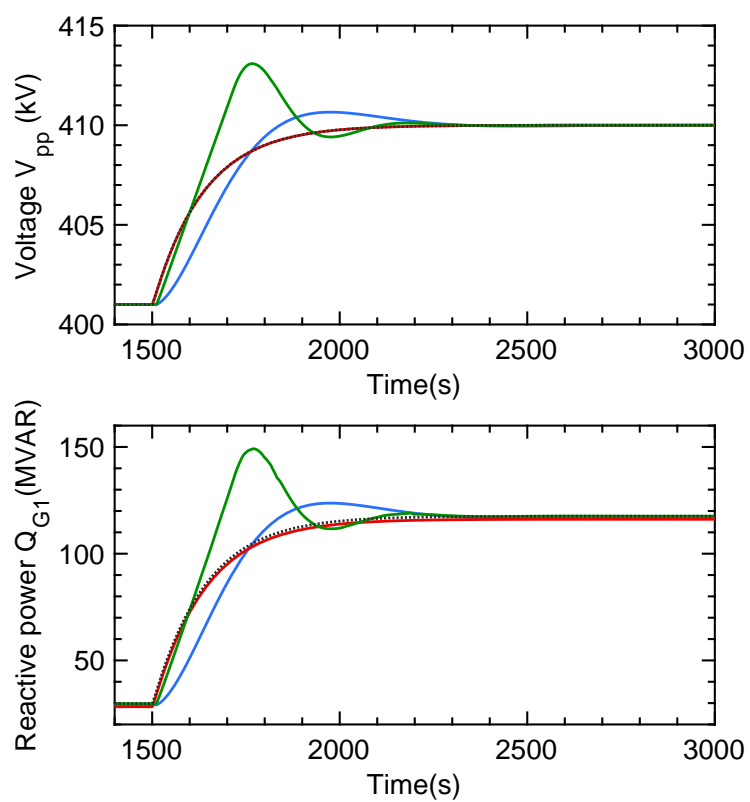

Figure 5: Impact of parametric variations $\Delta C_{v} / C_{q}$ on the SVC performance (blue : nominal classic SVC, red : nominal new SVC, green : disturbed classic SVC, black : disturbed new SVC).
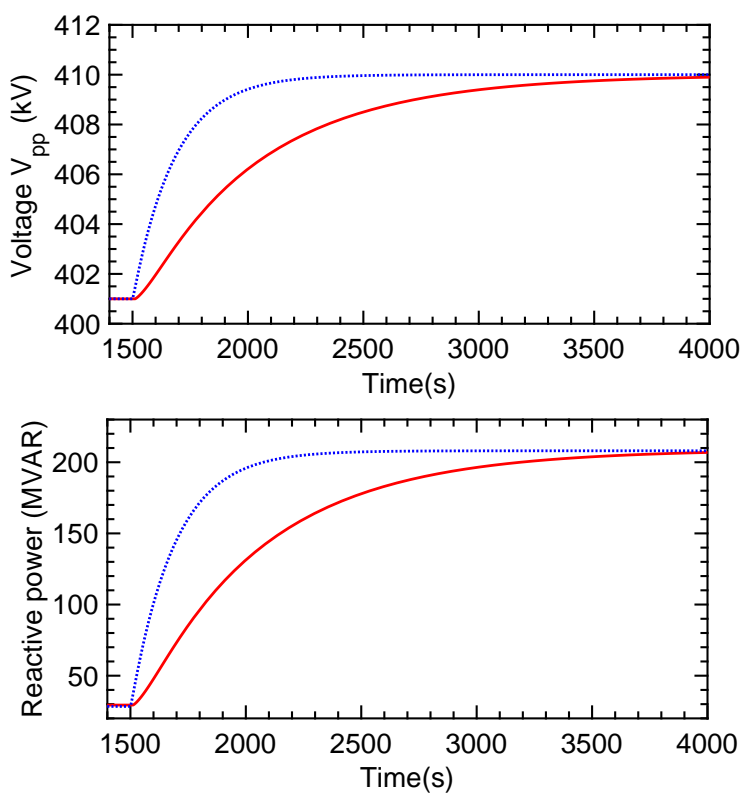

Figure 6: SVC performance in case of communication link failure (red : classic SVC, blue : new SVC). 


\section{B. Study 2 : parametric variations $\Delta C_{v} / C_{q}$}

To take into account of usual grid variations, the parameters of line 1 and 3 are multiplied by 2 and the parameters of line 2 and 4 are divided by 2 . These changes are taken into account only for simulation and not for the synthesis of the regulators. Figure 5 shows that the proposed controller maintain the required time-domain performances for the pilot-point voltage and the alignment is still achieved. However the classical SVC controller presents a large overshoot and the required time-domain performances are not respected in the presence of parametric variations.

\section{Study 2: Communication link failure}

Communications have a predominant role in close-loop performance. To validate this, resiliency to a single link failure between the power station 4 and the SVC is studied in figure 6. With the same control parameters as in previous study, the communication link from SVC to generator G4 is supposed to fail at $\mathrm{t}=1500 \mathrm{~s}$. This has no impact on performances of the loop closed with the new SVC controller. Nevertheless, system dynamic has significantly slowed down when using the classic SVC controller $(2500 \mathrm{~s}$ longer response)

\section{Study 3: Effects of delays}

So far, we have assumed that information exchanged between the power stations controllers is instantaneously available. However, in practice, it's not always the case. For this reason, in this study we examine the effects of transmission delays.
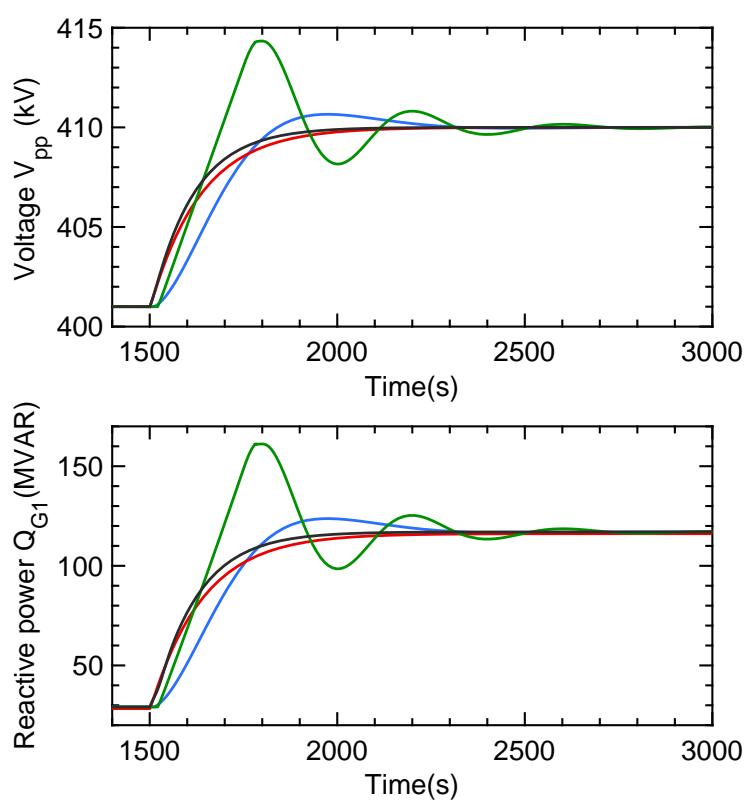

Figure 7: Impact of communication delays (pilot-point voltage and measured reactive power and controls) on the performance of SVC : nominal (blue: classic SVC, red: new SVC), scenario 1 (green: classic SVC, black: new SVC).
Table I: Communication delay

\begin{tabular}{|c|c|c|c|}
\hline & $V_{p p}$ & $Q_{i}$ & $u$ \\
\hline Scenario 1 & $10 s$ & $10 s$ & $10 s$ \\
\hline Scenario 2 & $20 s$ & $20 s$ & $20 s$ \\
\hline
\end{tabular}

1) Nominal/usual delays: As can be seen in figure 7, the proposed controller better tolerates the delays. At this low and usual level of delay, these response is not far from the nominal one. The classic one has large overshoots with small rise time. Moreover, if the communication delay increases, this can cause instability of the system as shown in the next section.

2) Delay stability margin: In this section, delays are pushed to larger values to estimate the stability margin. From figure 8 and Table I, it can be concluded that this margin is 20 $\mathrm{s}$ for the classic SVC and 50s for the new one. Notice also that even before instability, large overshoots which lead generators in reactive power generation limits are registered for classic SVC. This is avoided with the new SVC as seen in figure 8 .

\section{E. Study 4: Non minimal phase response}

The non minimal phase response of the classic SVC mentioned in Section II.A is now put into evidence in figure 9. At $t=1000 \mathrm{~s}$ the load is increased by 100 MVAR. As consequences; $V_{p p}$ drops and the reactive power of the generators $Q_{i}$ rapidly increases due to the electric dynamics.
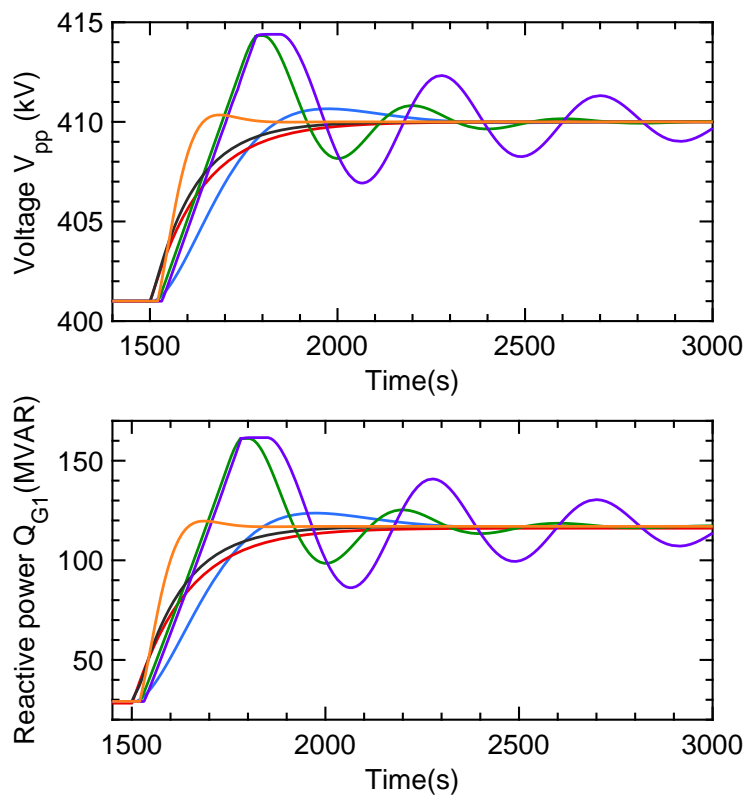

Figure 8: Impact of communication delay (measured pilot point voltage and controls) on the performance of secondary voltage controller: nominal (blue: classic SVC, red: new SVC), scenario 1 (green: classic SVC, black: new SVC), scenario 2 (purple: classic SVC, orange: new SVC). 
The reactive power loop 7 decreases the $Q_{i}$ to counteract this increase with respect to set-point $Q_{i}^{*}$. This leads to the overshoot in opposite direction in figure 9. After few seconds, the level $Q_{l e v}$ will be increased by equation 6 and this leads to the final increase of $Q_{i}$ to counteract the initial load increase. This phenomenon is due to the time scale separation of controls 7 and 8 it is well-known in automatic control. Same behaviour is registered for a generator outage $(t=2500 \mathrm{~s}$ in the same figure). It is clear from the same figure that the new controller avoids this phenomenon.

\section{CONCLUSION}

It has been shown that the problem of voltage control of a pilot-point and reactive power generation alignment of a given grid zone is dynamically feasible if an average level of reactive power is tracked instead of constant references; references given by a zone level based on voltage tracking. The outputs which can and should be tracked to set-points have been clearly identied. An optimal pole placement has been proposed. The resulting control is of multi-variable PI type and uses the same types of measures as the previous SVC schemes. It avoids the non minimal phase behavior of the local reactive power control loops and provides robustness properties (against parametric uncertainties and disturbances rejection). In comparison to CSVC, the implementation burden is lower since a stationary control is used instead of real-time optimization. No state estimation is used to implement the controller as it uses only variables which are available as measures. Robustness is improved against network topology changes, communication failures and uncertainties from the system and transmission delays.
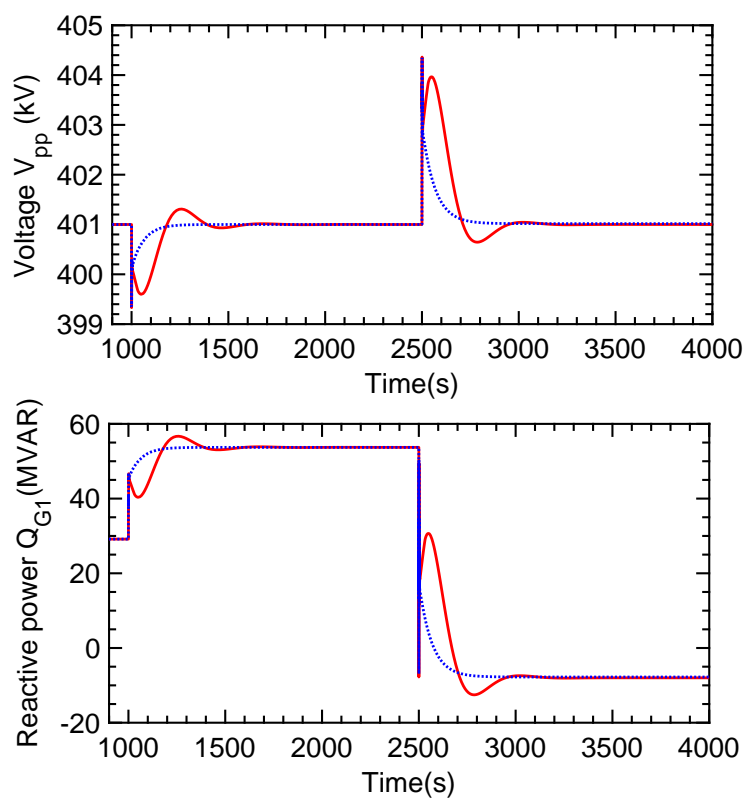

Figure 9: Non minimal phase response : 1-connexion of reactive load 100MVAR, 2- Generator 4 shutdown (red : classic SVC, blue : new SVC)
Future work will focus on extensions to the multiple pilotpoints case and implementation and validation on larger power systems zones. Choice of reactive powers to be included in the control will be optimized for dynamics performances improvement. Also, more advanced (and thus more robust) pole placement methods will be used for gains computation.

\section{REFERENCES}

[1] N. Martins, "The New CIGRE Task Force on Coordinated Voltage Control in Transmission Networks," IEEE Power Engineering Society Summer Meeting, pp. 305-306, 2000

[2] J. Y. Wen, Q. H. Wu, D. R. Turner, S. J. Cheng, and J. Fitch, "Optimal Coordinated Voltage Control for Power System Voltage Stability," IEEE Transactions on Power Systems, vol. 19, no. 2, pp. 1115-1122, 2004.

[3] H. Lefebvre, D. Fragnier, J. Y. Boussion, P. Mallet, and M. Bulot, "Secondary coordinated voltage control system: Feedback of EDF," Proceedings of the IEEE Power Engineering Society Transmission and Distribution Conference, vol. 1, pp. 290-295, 2000.

[4] S. Corsi, M. Pozzi, C. Sabelli, and A. Serrani, "The coordinated automatic voltage control of the Italian transmission grid - Part I: Reasons of the choice and overview of the consolidated hierarchical system," IEEE Transactions on Power Systems, vol. 19, no. 4, pp. 1723-1732, 2004.

[5] G. Taranto, N. Martins, D. Falcao, A. Martins, and M. dos Santos, "Benefits of applying secondary voltage control schemes to the Brazilian system," Proceedings of the IEEE Power Engineering Society Transmission and Distribution Conference, vol. 2, pp. 937942, 2000.

[6] G. Grigoras, B.-C. Neagu, F. Scarlatache, and R. C. Ciobanu, "Identification of pilot nodes for secondary voltage control using K-means clustering algorithm," 2017 IEEE 26th International Symposium on Industrial Electronics (ISIE), pp. 106-110, 2017.

[7] W. Gu, G. Lou, W. Tan, and X. Yuan, "A Nonlinear State EstimatorBased Decentralized Secondary Voltage Control Scheme for $\mathrm{Au}-$ tonomous Microgrids," IEEE Transactions on Power Systems, vol. 32 no. 6, pp. 1-1, 2017.

[8] N. M. Dehkordi, N. Sadati, and M. Hamzeh, "Distributed Robust Finite-Time Secondary Voltage and Frequency Control of Islanded Microgrids," IEEE Transactions on Power Systems, vol. 32, no. 5, pp. 3648-3659, 2017.

[9] M. Larsson, "Coordinated Voltage Control in Electric Power Systems," Ph.D. dissertation, Lund university, 2000.

[10] H. F. Wang, H. Li, and H. Chen, "Coordinated secondary voltage control to eliminate voltage violations in power system contingencies," IEEE Transactions on Power Systems, vol. 18, no. 2, pp. 588-595, 2003.

[11] B. Marinescu, "Robustness and coordination in voltage control of large-scale power systems," International Journal of Control, vol. 81, no. 10 , pp. $1568-1589,2008$

[12] F. Dong and B. H. Chowdhury, "Secondary voltage regulation for improved power plant reactive power coordination," Electric Power Components and Systems, vol. 35, no. 10, pp. 1181-1199, 2007.

[13] H.-Y. Su, F.-M. Kang, and C.-W. Liu, "Transmission Grid Secondary Voltage Control Method Using PMU Data," IEEE Transactions on Smart Grid, pp. 1-10, 2016.

[14] E. J. Davison and A. Goldenberg, "Robust Control of a General Servomechanism Problem: The Servo Compensator," Automatica, vol. 11, no. 4, pp. 461-471, 1975.

[15] B. A. Francis and W. M. Wonham, "The internal model principle of control theory," Automatica, vol. 12, no. 5, pp. 457-465, 1976.

[16] S. Skogestad and I. Postlethwaite, Multivariable feedback control: analysis and design. Wiley New York, 2007. 